\title{
Interactive comment on "Long-term variability of solar irradiance and its implications for photovoltaic power in West Africa” by Ina Neher et al.
}

\section{Anonymous Referee \#1}

Received and published: 17 June 2020

\section{General Comments}

This article looks at surface solar irradiance and photovoltaic power generation in West Africa. The authors use surface measurements of solar irradiance to evaluate satellitebased estimates of solar irradiance, which provide better temporal and spatial coverage. They then use the satellite-based estimate of solar surface irradiance to characterize its regional and temporal variability. Estimates of photovoltaic power yield are calculated as a simple linear function of the surface solar irradiance and again its spatial and temporal variability is characterized. 
The research described in this article is original, though the results are rather predictable based on the existing literature. Scientifically, I think the analysis is fairly sound, though I have some concerns about the implications of the errors in the satellite estimates of solar surface irradiance. The quality of the writing is reasonable. I have made some suggestions for improvements below.

Interactive

comment

1. The difference between the surface and SARAH estimates of $\mathrm{GHI}$ are rather large at two of the sites. Assuming that these errors are representative of the uncertainty in the SARAH product across the region, how does this impact on the subsequent analysis of spatial/temporal variability and trends (the errors are comparable to the scale of much of the spatial and temporal variabilities presented in section 5 and much larger than the total trend estimates). The section concludes "the evaluation shows that the SARAH-2.1 data record can be used to get a reasonable overview on the irradiance variability and trends to estimate the PV potential in West Africa". What magnitude errors would mean that the SARAH data record isn't suitable?

2. On a related note, I would be interested to see how the errors impact on the photovoltaic power yield estimates. How different would the estimates at the three surface sites be if you used the surface measured $\mathrm{GHI}$ rather than the satellite $\mathrm{GHI}$ as input?

3. I would consider changing the structure of the paper so that the description of the methodologies to calculate photovoltaic power yield (i.e section 3 ) is placed after the results for $\mathrm{GHI}$ and immediately before the presentation of the results for the photovoltaic power yield. 


\section{Specific Comments}

1. I'm not convinced the surface albedo shown in Fig 1(a) is particularly relevant for this study as it has no impact on the GHI. l'd suggest using a different image instead. Perhaps a snapshot visible image from SEVIRI?

2. The paragraph comparing MVIRI and SEVIRI (L100) seems incomplete. Which channels are used for SARAH? Which channels are on SEVIRI? When does SARAH use MVIRI and when SEVIRI?

3. Please can you specify what the MAE quoted for SARAH (L108) is measured against? Is this compared to surface-based observations? If so where and when?

4. I would move the text on ERA5 data (L117-120) to the following paragraph.

5. If I understand correctly, "b" in equation (5) represents the power required by the inverter, which is a function of temperature. Yet in table 3 , for $\mathrm{T}>35$ it has a positive value, which implies the inverter is generating power? Can you comment on this?

6. Why are some of the points in Figure 3 grey? Are these points where Delta AOD is negative?

7. For the trend analysis, can you take the uncertainty in the SARAH measurements into account in your estimates of the significance of the trend?

\section{Technical Corrections}

L25: I don't think "therewith" is the correct word here (nor in the other places where it is used in the article). 
L35: Remove total

L38: "sun zenith" should be "solar zenith"

L39: "shadings from the surrounding" should be "shade from the surroundings"

L98: "ground albedo" should be "surface albedo"

L160-L163: Are "a" and "b" the wrong way round here?

Interactive

comment

Fig. 2: Change legend to b1+a1, etc for consistency with text.

L196-199: Pair of sentences about SARAH performance $n$ Banizoumbou can be made more concise e.g. "In Banizoumbou, SARAH performance is consistent with previous evaluation of against BSRN stations".

L231: "temporally" should be "temporal"

L249: "periodes" should be periods

L250: "Mai" should be May

L269: "summer month" should be summer months

L272: change "are the most efficient modulator" to "have a large impact on"

L333: "providing" should be "demonstrating"

Interactive comment on Atmos. Chem. Phys. Discuss., https://doi.org/10.5194/acp-2020-306, 2020. 